\section{(2) OPEN ACCESS}

\title{
Comparison of intra-aneurysmal flow modification using optical flow imaging to evaluate the performance of Evolve and Pipeline flow diverting stents
}

\author{
Nicole Mariantonia Cancelliere, ${ }^{1}$ Patrick Nicholson, ${ }^{1}$ Ivan Radovanovic, ${ }^{2}$ \\ Karla Mirella Mendes, ${ }^{1}$ Emanuele Orru, ${ }^{1}$ Timo Krings, ${ }^{1,2}$ Vitor M Pereira ${ }^{1,2}$
}

\begin{abstract}
'Division of Neuroradiology, Department of Medical Imaging, Toronto Western Hospital, University Health Network Toronto, Ontario, Canada ${ }^{2}$ Division of Neurosurgery, Department of Surgery, Toronto Western Hospital, University Health Network, Toronto, Ontario, Canada
\end{abstract}

\section{Correspondence to} Dr Vitor M Pereira, Division of Neuroradiology, Department of Medical Imaging and Division of Neurosurgery, Department of Surgery, Toronto Western Hospital, Toronto, ON M5T 2S8, Canada; vitor.pereira@uhn.ca

Received 5 December 2019 Revised 23 January 2020 Accepted 27 January 2020 Published Online First 21 April 2020

\section{Check for updates}

(C) Author(s) (or their employer(s)) 2020. Re-use permitted under CC BY-NC. No commercial re-use. See rights and permissions. Published by BMJ.

To cite: Cancelliere NM Nicholson P, Radovanovic I, et al. J Neurolntervent Surg 2020:12:814-817.

\section{ABSTRACT}

Background Flow diverting stent (FDS) devices have revolutionized the treatment of large and complex brain aneurysms, but there is still room for improvement, particularly on the flow diversion properties and technical challenges associated with stent deployment. In this study we compared flow diversion properties between the new generation Surpass Evolve (Stryker) and the Pipeline Flex (Medtronic) devices by quantitatively evaluating intra-aneurysmal flow modification. Methods An in vitro experimental set-up was used, consisting of four patient-specific silicone models with internal carotid aneurysms and a circulating hemodynamic simulation system with pulsatile flow. The Evolve and Pipeline stents were deployed across the neck of each aneurysm model, in a randomized fashion, for a total of eight device deployments. A 60 frames/s digital subtraction angiography run was acquired before and after placement of each FDS. An optical flow-analysis method was used to measure intra-aneurysmal flow modification induced by the stent by calculating a mean aneurysm flow amplitude (MAFA) before and after stent placement and computing a ratio.

Results Average MAFA ratio values calculated from pre- and post-stent placement were significantly lower after deployment of the Evolve $(n=4$, mean $=0.62 \pm 0.09)$ compared with the Pipeline device $(n=4$,

mean $=0.71 \pm 0.06)(p=0.03)$.

Conclusions Our in vitro results show that the Evolve stent had a superior flow diversion effect compared with the Pipeline stent, which-based on clinical evidencesuggest it may promote faster aneurysm occlusion rates in patients.

\section{INTRODUCTION}

Flow diverting stent (FDS) devices are an effective treatment for large, giant and wide-neck intracranial aneurysms. Several different FDS devices are available today, which include, but are not limited to, the Pipeline (PED \& Flex; Medtronic), Surpass (Stryker), FRED (flow redirection endoluminal device, MicroVention), SILK (Balt Extrusion), and p64 (Phenox). Recent meta-analyses that evaluated the effectiveness of these FDS devices showed that the overall complete aneurysm occlusion rates were between $76-77.9 \%$ at 6 months follow-up, depending on the stents included. ${ }^{12}$ Although these occlusion rates are good, there is still room for improvement.

Much work is ongoing to improve flow diversion in an attempt to improve aneurysm treatment outcomes. Aneurysm flow diversion and occlusion rates have been shown to be positively correlated with a higher metal coverage ratio (MCR) (or lower stent porosity) ${ }^{3}$ and higher pore density ${ }^{4}$; however, as MCR increases so do the challenges of stent deployment, especially in tortuous anatomies and in giant or large aneurysms that require longer devices or telescoping reconstructions.

The first generation of flow diverters, including the Pipeline and Silk stents, were comprised of 48 wires made from chromium and cobalt or nitinol in different braided angles. The first iteration of the Surpass stent (Stryker, Fremont, CA) was the first FDS to use a higher wire density, varying from 48 to 96 wires. Although the increased MCR allowed for improved flow diversion and the initial clinical results were encouraging, it had a challenging stent mounted deployment system which affected navigation and deployment. ${ }^{5}$

The second generation of the Surpass stent, called the Surpass Evolve, has recently come to market and has a unique 64-wire design which overcomes some of the challenges with the earlier version of the Surpass system. By decreasing the wire thickness and maintaining the same MCR the stent is able to maintain flow diversion but improve navigability and ease of deployment. We hypothesize that the higher pore density and optimized braid angles of this novel device may improve its flow diversion properties compared with the Pipeline stent.

Optical flow digital subtraction angiography (DSA) imaging has been used to study the flow diversion properties of FDS both in vitro and in vivo. ${ }^{67}$ This method uses $3 \mathrm{D}$ rotational angiography (3D-RA) and DSA sequences with high temporal resolution to estimate blood flow velocities in the parent artery and aneurysm sac before and after FDS placement. Mean aneurysm flow amplitude (MAFA) ratio (MAFA-R) thresholds are clinically relevant as they have been shown to be independent predictors of aneurysm occlusion rates. ${ }^{6}$

The purpose of this study was to use optical flow DSA imaging, in a hemodynamically simulated and controlled in vitro set-up, in order to quantitatively 
compare the effectiveness of aneurysm flow diversion between the new Surpass Evolve (64-wire design) and the well-studied Pipeline Flex (48-wire design) FDS.

\section{MATERIALS AND METHODS In vitro set-up}

The study was performed using four patient-specific silicone models of various internal carotid aneurysms. Segmentation of the vessel lumen was performed using 3D-RA imaging and silicone models were created using a 3D-printed lost wax method (Elastrat, Geneva, Switzerland). Each model had one inlet (internal carotid artery (ICA)) and two outlets (middle and anterior cerebral arteries).

The models were connected to a hemodynamic simulation system compris a circulating water bath connected to an adjustable steady pump (Cole Parmer, Vernon Hills, Illinois) set to a mean volumetric flow rate of $3.96 \mathrm{~mL} / \mathrm{s}$. A software program (LabView, National Instruments, Austin, TX) and data acquisition card (DAQ, National Instruments, Austin, TX) were used to introduce a $1 \mathrm{~Hz}$ pulsatile volumetric flow mimicking that of the ICA waveform, similar to previous in vitro flow studies. ${ }^{8}$ Rigid tubing was used to preserve pulsatility of flow.

\section{FDS implantation}

Following 3D-RA of the flow model, optimal working projections were selected. Using a tri-axial support system under fluoroscopic guidance (AXS Infinity Sheath 0.088 inch + AXS Catalyst 5 DAC 0.058 inch + Excelsior XT-27 microcatheter 0.036 inch; Stryker, Fremont, CA), either an Evolve or Pipeline $(4.25 \times 25 \mathrm{~mm})$ stent was fully deployed in the silicone model, in a randomized fashion, by an experienced interventional neuroradiologist. The device was then removed from the model and the other stent was deployed. Flow measurements were acquired before and after each stent implantation. This was repeated for four separate aneurysm models ( $n=8$ implantations).

Device specifications regarding the 64-wire (Surpass Evolve, Stryker, Fremont, CA) and 48-wire (Pipeline Flex, Medtronic, Irvine, CA) FDS are summarized in table 1. A DSA run and/ or Vaso-CT (Philips Healthcare, Best, the Netherlands) were performed to confirm suitable stent apposition to the silicone vessel wall. Balloon angioplasty was performed to improve wall apposition if needed.

\section{Intra-aneurysmal flow analysis}

After each aneurysm model was connected to the pulsatile hemodynamic simulation system, a 3D-RA was acquired. From this, optimal X-ray tube projections for MAFA analysis were selected to minimize superimposition of vascular anatomy over the aneurysm sac and to visualize the parent artery in profile in order to obtain accurate flow measurements. A 60 frames/s DSA run was acquired before and after FDS placement during a $1 \mathrm{~mL} / \mathrm{s}$ injection of iodinated contrast media (Omnipaque/iohexol, $300 \mathrm{mgI} /$

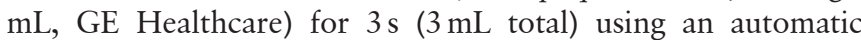
injector pump (Medrad, Bayer Healthcare) for consistent results.

Radiographic information from the 3D-RA and two DSA runs were loaded into the flow analysis software on the Philips Healthcare interventional workstation (AneurysmFlow, Xtravision workstation release V8.8.1). The software application calculates the flow within the aneurysm sac by applying an optical flow algorithm on the DSA time series. By tracking the spatial and temporal changes in contrast density between successive images of the run, the software is able to produce vectors referred to as detector velocity fields (DVFs). DVFs contain both magnitude and direction in 2D space, orthogonal to the projection plane, and are visualized using short streamlines superimposed on a magnitude color map.

An experienced technologist manually defined the pizels of interest on each dataset and drew a region of interest (ROI) around the aneurysm sac with attention to clearly defining the aneurysm neck and excluding superimposed vessels with high flow, if applicable. A time and spatial average of the DVFs was computed to produce a MAFA. MAFA-R was then computed for each stent by dividing the MAFA ${ }_{\text {post }}$ MAFA $_{\text {pre }}$. A paired student's t-test with a threshold for $\mathrm{p}<0.05$ was used to determine statistical significance.

\section{RESULTS}

\section{Stent implantations}

Navigation and implantation was successful for all eight devices. Good wall apposition was achieved in all cases, confirmed either by a DSA run and/or 3D Vaso-CT imaging. One case, model $\mathrm{D}$, required balloon angioplasty to improve stent apposition for both devices (figure 1).

\section{MAFA analysis}

Aneurysm flow amplitude was calculated successfully in all cases. Figure 2 shows a comparison between MAFA analyzed before

Table 1 Comparison between device specifications for Surpass Evolve and Pipeline Flex flow diverting stents

\begin{tabular}{lll}
\hline & Evolve & Pipeline \\
\hline Design & & \\
& & \\
& 64 & \\
No. of wires & Braided & 48 \\
Type & Higher & Braided \\
Braid angle & Higher & Lower \\
Pore density & Thinner & Lower \\
Wire thickness & Similar & Thicker \\
Metal coverage & $80 \%$ cobalt chromium / & Similar \\
Composition & $20 \%$ platinum tungsten & $75 \%$ cobalt chromium / \\
& & $25 \%$ platinum tungsten \\
\hline
\end{tabular}




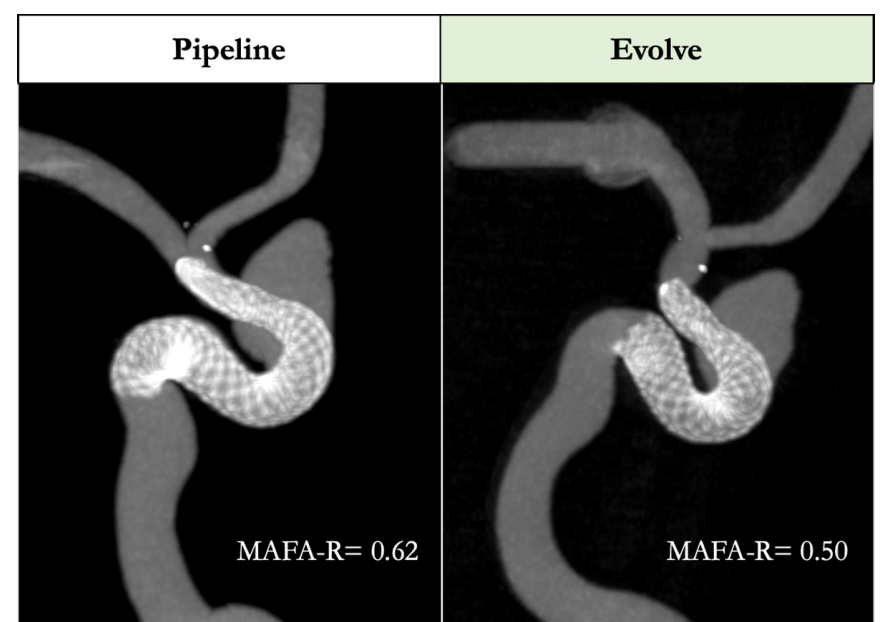

Figure 1 Representative views of 3D Vaso-CT imaging demonstrating wall opposition after angioplasty of both a Pipeline Flex $(4.25 \times 25 \mathrm{~mm})$ and a Surpass Evolve $(4.25 \times 25 \mathrm{~mm})$ stent, sequentially placed in the same patient-specific silicone aneurysm model. MAFA- $R$, mean aneurysm flow amplitude ratio.

and after Pipeline and Evolve FDS were placed in a paraclinoid aneurysm. In terms of MAFA-R changes, Evolve performed superiorly to Pipeline, causing a greater reduction in flow within the aneurysm quantified by a lower MAFA-R and visualized by a transition from red to white within the blue ROI on the velocity magnitude color map.

Intra-aneurysmal flow changes induced by each FDS in the four different aneurysm models are summarized in table 2. Average aneurysm flow amplitude was significantly lower after implantation of the Evolve stent (mean MAFA 0.62 \pm 0.09 ) compared with the Pipeline device (mean MAFA 0.71 \pm 0.06$)(\mathrm{p}=0.03)$.

\section{DISCUSSION}

\section{Are 64 wires better than 48 ?}

Using novel optical flow techniques and a controlled in vitro setup, our study has shown that the new generation 64-wire Surpass Evolve stent has superior flow diversion efficacy over the traditional 48-wire Pipeline Flex stent. MAFA-Rs compared before and after stent placement in four patient-specific ICA aneurysm models were consistently and significantly lower after placing an

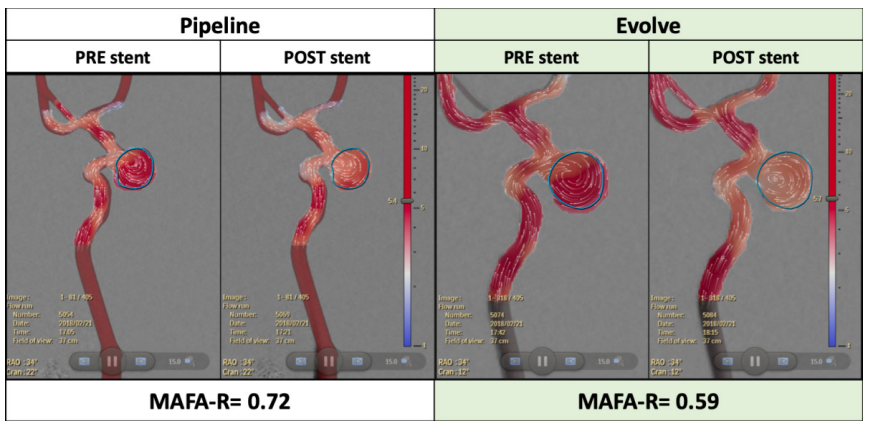

Figure 2 Representative mean aneurysm flow amplitude analysis displaying averaged detector velocity fields (white dots with lines representing the direction of travel) superimposed on a velocity magnitude color $\operatorname{map}\left(\mathrm{m}^{2} / \mathrm{s}\right.$; blue=slower, red=faster) for each flow diverting stent, before and after stent placement. Aneurysm region of interest highlighted in blue. MAFA-R, mean aneurysm flow amplitude ratio.
Table 2 Summary of aneurysm flow ratios between Evolve and Pipeline devices. Mean aneurysmal flow amplitude ratio values within the aneurysm sac, calculated from pre- and post Evolve and Pipeline deployment, were compared in four patient specific silicone models

\begin{tabular}{|c|c|c|c|c|c|c|}
\hline \multirow[b]{2}{*}{ Model } & \multicolumn{3}{|l|}{ Evolve } & \multicolumn{3}{|l|}{ Pipeline } \\
\hline & MAFA $_{\text {pre }}$ & MAFA $_{\text {post }}$ & Ratio & MAFA $_{\text {pre }}$ & MAFA $_{\text {post }}$ & Ratio \\
\hline A & 2.4 & 1.6 & 0.67 & 1.9 & 1.4 & 0.74 \\
\hline B & 4.1 & 2.4 & 0.59 & 3.9 & 2.8 & 0.72 \\
\hline C & 3.4 & 2.4 & 0.71 & 2.3 & 1.7 & 0.74 \\
\hline D & 4.8 & 2.4 & 0.50 & 6.3 & 3.9 & 0.62 \\
\hline Average & & & 0.62 & & & 0.71 \\
\hline$S D$ & & & \pm 0.09 & & & \pm 0.06 \\
\hline
\end{tabular}

MAFA, mean aneurysmal flow amplitude.

identical stent in the same patient-specific aneurysm model, with 64-wire FDS decreasing the flow to produce a MAFA-R on average of 0.62 and the 48 -wire devices producing a MAFA-R of only 0.71 . We hypothesize that this improvement in flow diversion may be attributed to a combination of the higher braid angles and greater pore density of the Evolve stent design.

Previous clinical studies have shown that the MAFA-R threshold is an independent predictor of aneurysm thrombosis. ${ }^{6}$ When larger ICA aneurysms are considered, such as those included in our study, MAFA-R thresholds of 0.89 and 0.64 have been shown to predict a high probability of complete aneurysm occlusion at 12 months and 3 months, respectively. ${ }^{9}$ Based on these findings, we predict that Evolve may promote faster aneurysm occlusion rates than Pipeline since the average MAFA-R for Evolve (0.62) fell below the 3 month occlusion threshold, whereas the average MAFA-R for Pipeline (0.71) was above this threshold.

To date, there are limited quantified methods to predict efficacy of treatment with FDS. Clinical decisions are typically guided by visual cues on DSA, such as contrast agent stagnation or partial filling signs, despite the lack of clinical evidence to support this as an independent predictor of aneurysm occlusion. ${ }^{6}$ Another group has recently published a study that analyzed convection and contrast diffusion to demonstrate that Evolve has greater in vitro flow diversion effects than Pipeline, ${ }^{10}$ similar to our findings. However, our study is the first to investigate the flow diversion properties of Evolve using a validated advanced DSA imaging optical flow quantification method. Optical flow imaging has been used to investigate flow diversion for various neurovascular devices in the past, such as stents to assist coiling ${ }^{8}$ or intrasaccular devices ${ }^{11}$; however, to our knowledge, our study is the first to use the tool to investigate FDS in an in vitro setting.

\section{Limitations}

Although great effort was made to mimic a realistic hemodynamic environment by using patient specific aneurysm models and ICA pulsatile waveforms, various in vivo characteristics, such as aneurysm wall properties or patient specific flow rates, were unknown and therefore were not possible to control for. The changes in flow diversion properties associated with the newer stent types will need further investigation in patients. Similarly, further studies should be performed to elucidate the relationship between MAFA-R calculated in models versus in patients. Additionally, our study was limited to eight stent deployments. A larger sample size to include aneurysms in different locations beyond the ICA could also be of interest. 


\section{CONCLUSIONS}

Our study suggests that a new FDS, Surpass Evolve, designed with 64 braided wires, has superior flow diversion effects compared with the traditional 48-wire Pipeline Flex design. This improved flow diversion may result in faster and better aneurysm occlusion rates for patients treated with this new generation of FDS.

Contributors All authors were substantially involved in the study design and interpretation, as well as manuscript revision and final approval. NMC and VMP were involved in the conception of the study, acquisition and analysis of the data, and initial manuscript draft.

Funding The authors have not declared a specific grant for this research from any funding agency in the public, commercial or not-for-profit sectors.

Competing interests VMP is a consultant for Stryker Neurovascular and Medtronic Neurovascular.

Patient consent for publication Not required.

Provenance and peer review Not commissioned; externally peer reviewed.

Data availability statement Data are available upon reasonable request; please contact vitor.pereira@uhn.ca.

Open access This is an open access article distributed in accordance with the Creative Commons Attribution Non Commercial (CC BY-NC 4.0) license, which permits others to distribute, remix, adapt, build upon this work non-commercially, and license their derivative works on different terms, provided the original work is properly cited, appropriate credit is given, any changes made indicated, and the use is non-commercial. See: http://creativecommons.org/licenses/by-nc/4.0/.

\section{REFERENCES}

1 Ye G, Zhang M, Deng L, et al. Meta-analysis of the efficiency and prognosis of intracranial aneurysm treated with flow diverter devices. J Mol Neurosci 2016;59:158-67.

2 Brinjikji W, Murad MH, Lanzino G, et al. Endovascular treatment of intracranial aneurysms with flow diverters: a meta-analysis. Stroke 2013;44:442-7.

3 Jou L-D, Chintalapani G, Mawad ME. Metal coverage ratio of pipeline embolization device for treatment of unruptured aneurysms: reality check. Interv Neuroradiol 2016;22:42-8.

4 Sadasivan C, Cesar L, Seong J, et al. An original flow diversion device for the treatment of intracranial aneurysms: evaluation in the rabbit elastase-induced model. Stroke 2009;40:952-8.

5 De Vries J, Boogaarts J, Van Norden A, et al. New generation of Flow Diverter (surpass) for unruptured intracranial aneurysms: a prospective single-center study in 37 patients. Stroke 2013;44:1567-77.

6 Pereira VM, Bonnefous 0 , Ouared R, et al. A DSA-based method using contrastmotion estimation for the assessment of the intra-aneurysmal flow changes induced by flow-diverter stents. AJNR Am J Neuroradiol 2013;34:808-15.

7 Bonnefous O, Pereira VM, Ouared R, et al. Quantification of arterial flow using digital subtraction angiography. Med Phys 2012;39:6264-75.

8 Pérez MA, Henkes H, Bouillot P, et al. Intra-aneurysmal hemodynamics: evaluation of pCONus and pCANvas bifurcation aneurysm devices using DSA optical flow imaging. J Neurointerv Surg 2016;8:1197-201.

9 Pereira M V, Brina O, Bracken J, et al. E-081 assessment of the MAFA ratio as a quantitative prognostic marker of aneurysm occlusion after flow diverter treatment. J Neurolnterv Surg 2016;8:A84-5.

10 Sadasivan C, Fiorella D. Preliminary in vitro angiographic comparison of the flow diversion behavior of Evolve and Pipeline devices. J Neurointerv Surg 2019. doi:10.1136/neurintsurg-2019-015455. [Epub ahead of print: 13 Nov 2019].

11 Frölich AM, Nawka MT, Ernst M, et al. Intra-aneurysmal flow disruption after implantation of the Medina ${ }^{\circledR}$ embolization device depends on aneurysm neck coverage. PLoS One 2018;13:e0191975. 